\title{
Modeling phase transitions in rapidly expanding systems
}

\section{Igor Mishustin*}

Frankfurt Institute for Advanced Studies, Ruth-Moufang Str. 1, D-60438 Frankfurt am Main, Germany

Kurchatov Institute, Russian Research Center, 123182 Moscow, Russia

E-mail: mishustinefias.uni-frankfurt.de

In this talk I first discus hydrodynamical simulations of relativistic heavy-ion collisions within the lab energy domain of (5-40) AGeV. The calculations are done within the $3 \mathrm{~d}$ one-fluid model starting with cold nuclei in the initial state. Parameters of the compressed zone and matter trajectories in the temperature-density plane are calculated for two cases, with and without the deconfinement phase transition. An interesting observation is that in the equilibrium scenario the parameters of the final hadronic state are insensitive to the presence of the phase transition at early times. Then I consider the possibility of explosive hadronization where the quark-gluon plasma first disintegrates into droplets which later on decay into hadrons. This scenario is quite natural in the case of the first order phase transition when the spinodal instability drives the system into the inhomogeneous droplet phase. I present simple estimates for the droplet size based on the energy balance between the collective expansion energy and the surface energy. It is interesting that the fragmentation of the quark-gluon phase is predicted also for the crossover-type transition if the bulk viscosity becomes large in the transition region. In both cases the characteristic droplet size should decrease with increasing the collective expansion rate. Finally I will discuss possible observable signatures of quark-gluon droplets such as strong non-statistical fluctuations of hadron multiplicities in momentum space. These predictions can be checked in the energy scan program at RHIC, as well as in the future FAIR and NICA experiments.

5th International Workshop on Critical Point and Onset of Deconfinement - CPOD 2009,

June 08 - 122009

Brookhaven National Laboratory, Long Island, New York, USA

\footnotetext{
*Speaker.
} 


\section{Introduction}

High-energy heavy-ion collisions provide a unique tool for studying properties of hot and dense strongly-interacting matter in the laboratory. The theoretical description of such collisions is often done within the framework of a hydrodynamic approach. This approach opens the possibility to study the sensitivity of collision dynamics and secondary particle distributions to the Equation of State (EOS) of the produced matter. In this way one can get information about the deconfinement phase transition at intermediate stages of the collision process when the temperature and/or baryon density become high enough. The problem is however that many traces of the deconfinement phase transition may be erased during the hadronization process. Therefore, detailed dynamical simulations and comparisons with experimental data are needed to find convincing evidences of this phase transition.

\section{Equation of state with first order phase transition}

In our hydrodynamical simulations we use an EOS derived recently in ref. [1]. which includes the Hadron Gas (HG) at low energy densities. the Quark-Gluon Plasma (QGP) at high energy densities and a Mixed Phase (MP) in between. In the hadronic phase we include contributions of lightest hadrons with masses $m_{i} \lesssim 2 \mathrm{GeV}$, altogether 59 mesonic and 41 baryonic species listed in [2]. This corresponds to 307 different isospin states of mesons, baryons and antibaryons. In these calculations we do not include a very broad scalar meson resonance $f_{0}(600)$ with mass $m \sim$ $0.6 \mathrm{GeV}$ and width $\Gamma \gtrsim 0.6 \mathrm{GeV}$. Except of this state a very similar set of hadrons has been used in the THERMUS thermal model [3]. For the quark-gluon phase we use the EOS of the MIT bag model including a perturbative correction due to the chromomagnetic interaction as in ref. [4]. Properties of the MP were determined by applying the Gibbs criteria for the system characterized by two chemical potentials, responsible for the baryon number and strangeness conservation. The condition of zero net strangeness was imposed.

It should be emphasized that using different models for describing different phases may lead either to the first order phase transition or no phase transition at all. In our calculations we have faced the latter situation when considering ideal multi-species hadron gas. To obtain a reasonable phase diagram, e.g. as shown in Fig. 1, we were forced to introduce a finite volume of hadrons, $v \simeq$ $1 \mathrm{fm}^{3}$. The thermodynamical functions were calculated within the excluded volume approximation following the method of ref. [5]. Results of these calculations are shown in Figs. 1 and 2. As expected, we obtain a first order deconfinement phase transition between the HG and the QGP. The phase transition is rather week at small baryon chemical potentials $\mu$ but becomes stronger at larger values of $\mu$. The critical temperature $T_{c}=165 \mathrm{MeV}$ at $\mu=0$ was obtained by a proper choice of the bag constant.

The figures also show the adiabatic trajectories for several values of entropy per baryon S/B ranging from 5 to 300 . It is interesting to note that the temperature grows when going through the mixed phase from the QGP to the HG phase. This effect was first noticed in ref. [7]. But such behavior is not universal, e.g. chiral models like linear sigma model or NJL model demonstrate the opposite trend [8]. 


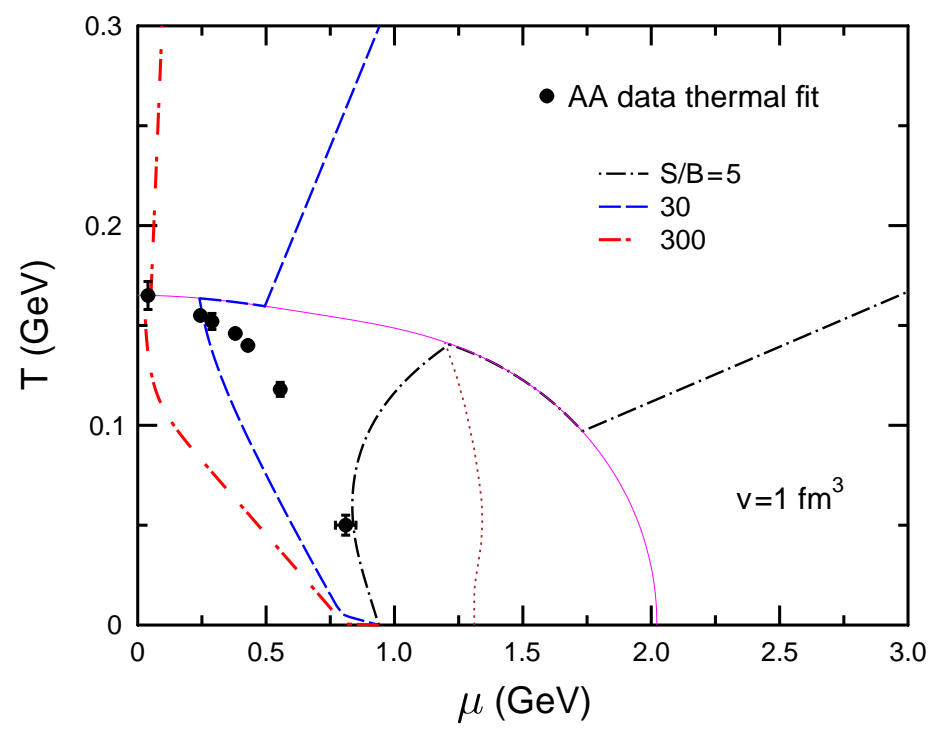

Figure 1: Phase diagram of strongly interacting matter in the $\mu-T$ plane constructed in ref. [1] (see the text). The solid line represents the phase transition boundary. The dashed and dash-dotted lines show isentropic trajectories for different values of entropy per baryon. Full dots correspond to the $\mu, T$ values obtained from thermal fits of hadron yields [6] observed in central $\mathrm{Au}+\mathrm{Au}$ and $\mathrm{Pb}+\mathrm{Pb}$ collisions at different bombarding energies. The region between the dotted and solid lines contains acausal states with $c_{s}>1$.

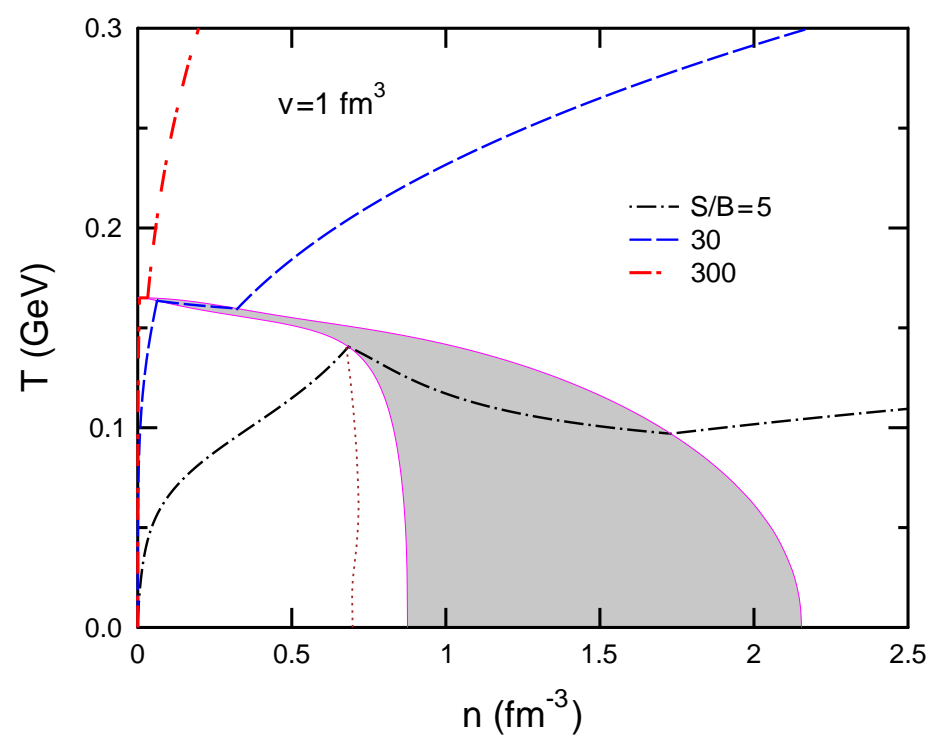

Figure 2: Boundaries of different phases and adiabatic trajectories in the $n-T$ plane $\left(v=1 \mathrm{fm}^{3}\right)$. The shaded area shows the mixed phase region. The hadronic states on the right from the dotted line have sound velocities $c_{s}>1$. 

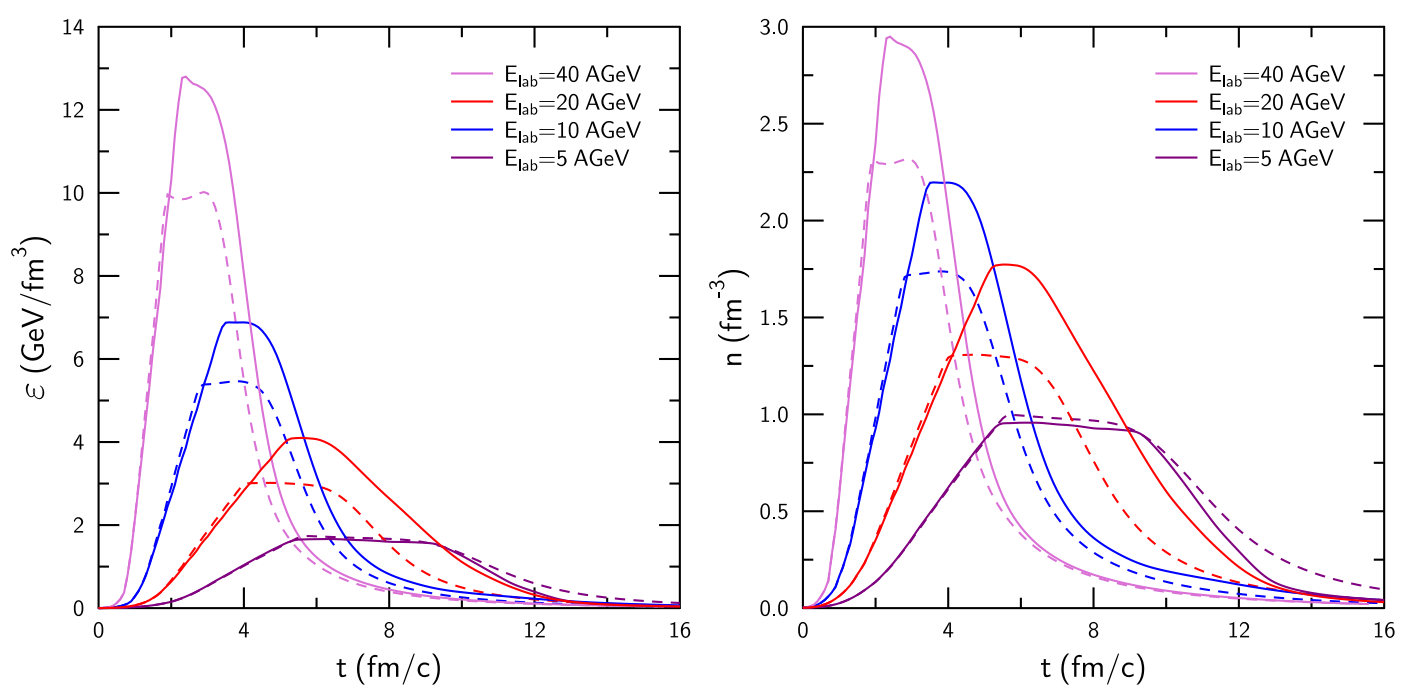

Figure 3: Time dependence of the energy density (left) and the baryon density (right) of the fluid in the central box as predicted by the $3 \mathrm{~d}$ hydro simulations for central $\mathrm{Au}+\mathrm{Au}$ collisions at different bombarding energies (indicated in the figure). Full lines and dashed lines correspond to the EOS with and without the deconfinement phase transition.

\section{3d hydrodynamical simulations of nuclear collisions}

The hydrodynamic modeling of relativistic nuclear collisions has a long history. The most popular simple versions of this approach were proposed by Landau [9] and Bjorken [10]. To study the matter evolution in the course of a relativistic heavy-ion collision we use a full $3 \mathrm{~d}$ version of the perfect-fluid dynamical model developed by the Frankfurt group and employing the SHASTA algorithm [11]. The numerical aspects and detailed results of this work will be described in ref. [12]. Here we present only a few results of numerical simulations obtained for two equations of state, EOS-PT and EOS-HG, where the first one corresponds to the hadron resonance gas and the second one includes the deconfinement phase transition.

Figure 3 shows the time evolution of the energy density and baryon density in central $\mathrm{Au}+\mathrm{Au}$ collisions at different bombarding energies. Their values are calculated in the central box with dimensions $\left(2 \times 2 \times 2 / \gamma_{\mathrm{cm}}\right) \mathrm{fm}^{3}$. One can see that the threshold energy to reach the pure QGP phase (energy density above $2 \mathrm{GeV} / \mathrm{fm}^{3}$ ) is about $5 \mathrm{AGeV}$. Comparison of calculations with different EOS shows that with the phase transition the matter reaches higher energy and baryon densities as compared with the pure hadronic phase. The corresponding maximum values range from 2 to 13 $\mathrm{GeV} / \mathrm{fm}^{3}$ and from 1 to $3 \mathrm{fm}^{-3}$ when bombarding energy increases from 5 to $40 \mathrm{AGeV}$.

More clear information about the states of matter in central box can be extracted from Fig. 4 showing the matter trajectories in the $T-\mu$ plane. One can see that the initial heating and compression of matter is very fast, so that the QGP phase is reached within less than $1 \mathrm{fm} / \mathrm{c}$ after the first contact of nuclei. At $10 \mathrm{AGeV}$ the system spends abut $4 \mathrm{fm} / \mathrm{c}$ in the QGP phase and then about $3 \mathrm{fm} / \mathrm{c}$ in the mixed phase before returning into the hadronic phase. At $40 \mathrm{AGeV}$ the time spent in the QGP phase is somewhat longer. In any case these times are very short, so that the 

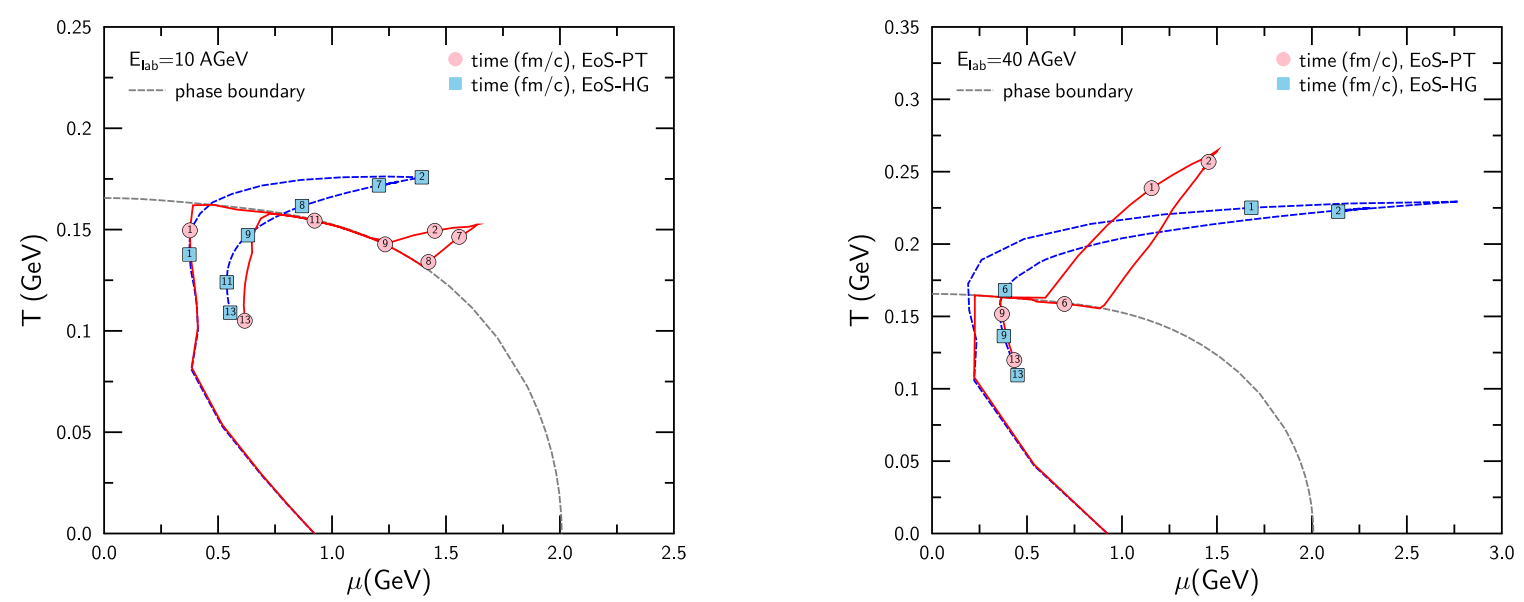

Figure 4: Evolution of temperature and chemical potential of the fluid in the central box as predicted by the $3 \mathrm{~d}$ hydro calculations for central $\mathrm{Au}+\mathrm{Au}$ collisions at $\mathrm{E}_{\mathrm{lab}}=10$ (left) and 40 (right) AGeV. Results are shown for two equations of state: with (full line) and without (dashed line) the phase transition.

transformation of the QGP into the hadron gas may not follow the equilibrium scenario. in the next Section I will consider an alternative mechanism. Here I want only to point out one more problem associated with the equilibrium scenario: besides of a short delay of about $1 \mathrm{fm} / \mathrm{c}$ the final states of the system are very similar in calculations with and without the phase transition. This means that the final spectra of produced hadrons are not sensitive to the intermediate stages of the reaction where the deconfinement-hadronization transition was taking place.

\section{Explosive hadronization}

Let us consider now a simplified picture where the system expands according to the Hubble law, $v(r)=H \cdot r$, where $v$ is the local collective velocity and $H$ is a function of time, as e.g. $H \propto 1 / t$, in the Bjorken model.

As demonstrated in refs. [13, 14], in a rapidly expanding system a first order phase transition will not follow the equilibrium phase coexistence trajectory as predicted by the hydrodynamic model (see Fig. 4). The formation of the mixed phase will be hindered by the potential barrier separating two competing phases. Instead, the high-temperature phase will expand further, until it enters the spinodal region where $c_{s}^{2}<0$ and the existence of the uniform phase becomes impossible. Then, due to intrinsic instabilities it will disintegrate into droplets surrounded by the undersaturated low-temperature phase. Different aspects of spinodal decomposition in expanding systems were discussed in refs. [15, 16, 17]. For clarity, below we use capital letters Q and H (not to be confused with the Hubble constant $H$ ) for the deconfined quark-gluon phase and the hadronic phase, respectively.

Following this picture, let us assume that the dynamical fragmentation of the deconfined phase has resulted in a collection of $\mathrm{Q}$ droplets embedded in a dilute $\mathrm{H}$ phase, as illustrated in Fig. 6. The 
optimal droplet size can be determined by applying a simple energy balance argument saying that the droplets are formed when the collective kinetic energy within the individual droplet is equal to its surface energy, $E_{\text {kin }}(R)=E_{\text {surf }}(R)$, where

$$
E_{\mathrm{kin}}(R)=\frac{1}{2} \int_{0}^{R} \Delta \mathscr{E}[v(r)]^{2} 4 \pi r^{2} d r=\frac{2 \pi}{5} \Delta \mathscr{E} H^{2} R^{5},
$$

and $E_{\text {surf }}(R)=4 \pi R^{2} \gamma$, where $\Delta \mathscr{E}=\mathscr{E}_{\mathrm{Q}}-\mathscr{E}_{\mathrm{H}}$ is the energy density difference of the two phases, and $\gamma$ is the corresponding surface tension. Then the optimal droplet radius is obtained as

$$
R^{*}=\left(\frac{10 \gamma}{\Delta \mathscr{E} H^{2}}\right)^{1 / 3}
$$

As eq. (4.2) indicates, the droplet size depends strongly on $H$. When expansion is slow (small $H$ ) the droplets are big. In the adiabatic limit the process may look like a fission of a cloud of plasma. But fast expansion should lead to very small droplets. This state of matter is very far from a thermodynamically equilibrated mixed phase, particularly because the droplet size is determined by the expansion rate but not by the thermodynamics alone. One can say that the metastable $\mathrm{Q}$ matter is torn apart by a mechanical strain associated with the collective expansion.

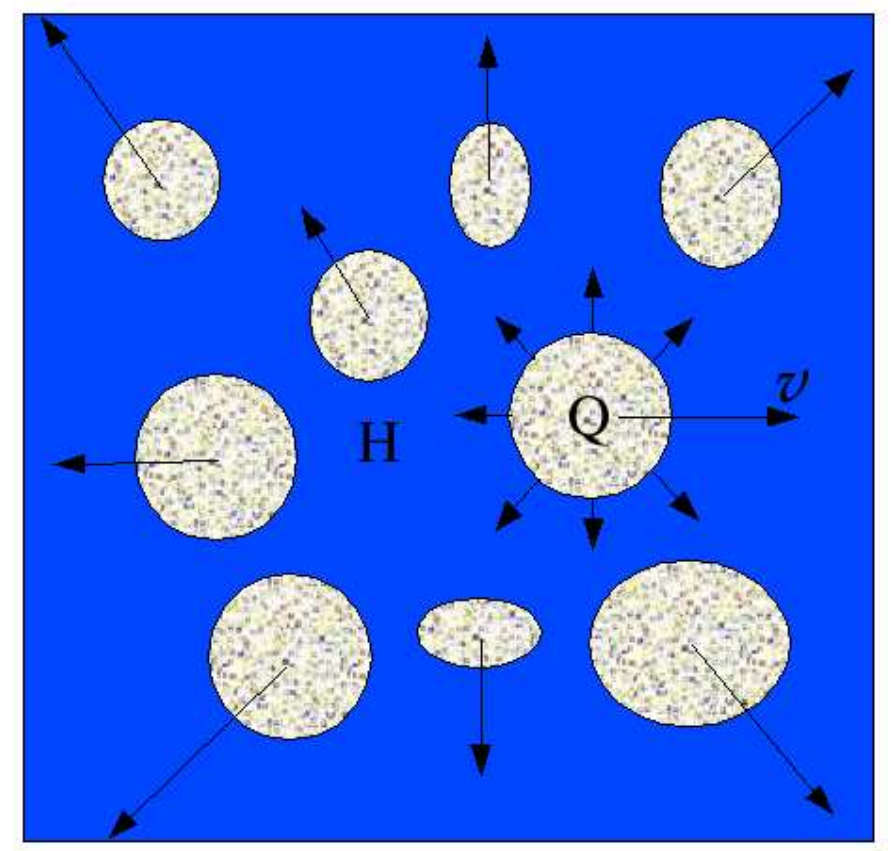

Figure 5: (Color online) Schematic view of multi-droplet state produced after the dynamical fragmentation of a unstable high energy-density $\mathrm{Q}$ phase. The $\mathrm{Q}$ droplets are embedded in the low energy-density $\mathrm{H}$ phase. Each droplet emits hadrons as a thermal source, as well as participates in the overall Hubble-like expansion.

In numerical estimates I consider two cases: $H^{-1}=2 \mathrm{fm} / \mathrm{c}$, i.e. fast expansion associated with nuclear collisions at top SPS and RHIC energies, and $H^{-1}=6 \mathrm{fm} / \mathrm{c}$, i.e. slow expansion associated 
with the low SPS and future FAIR-NICA energy domain. One should also specify two other parameters, $\gamma$ and $\Delta \mathscr{E}$. The surface tension $\gamma$ is a subject of debate at present. Lattice simulations indicate that it could be as low as a few $\mathrm{MeV} / \mathrm{fm}^{2}$ in the vicinity of the critical point. However, for our nonequilibrium scenario, more appropriate values may be closer to $10-20 \mathrm{MeV} / \mathrm{fm}^{2}$, which follow from effective chiral models. So, let us take $\gamma=10 \mathrm{MeV} / \mathrm{fm}^{2}$ for rough estimates. Bearing in mind that nucleons and heavy mesons are the smallest Q droplets, one can take $\Delta \mathscr{E}=0.5 \mathrm{GeV} / \mathrm{fm}^{3}$, i.e. the energy density inside the nucleon. Then one gets $R^{*}=0.9 \mathrm{fm}$ for $H^{-1}=2 \mathrm{fm} / \mathrm{c}$ and $R^{*}=1.9 \mathrm{fm}$ for $H^{-1}=6 \mathrm{fm} / \mathrm{c}$. As follows from eq. (4.2), for a spherical droplet $V \propto 1 / \Delta \mathscr{E}$, and in the first approximation its mass,

$$
M^{*} \approx \Delta \mathscr{E} V=\frac{40 \pi}{3} \frac{\gamma}{H^{2}},
$$

is independent of $\Delta \mathscr{E}$. For the two values of $R^{*}$ given above the optimal droplet mass is $\sim 1.7 \mathrm{GeV}$ and $\sim 15 \mathrm{GeV}$, respectively.

It is interesting that in the first case the droplet size and mass are in the range of typical hadron parameters. This means that in a very fast expansion the QGP phase splits into minimalsize droplets, i.e. hadrons and hadronic resonances. Nevertheless, this is not a standard phase transition but a direct conversion of the overcooled and overstretched QGP phase into hadrons, without going through the mixed phase. As argued in ref. [18], this mechanism can naturally explain such interesting observations as constituent quark scaling in elliptic flow and enhanced production of multi-strange baryons.

However, in the case of $H^{-1}=6 \mathrm{fm} / \mathrm{c}$ the droplet size and mass are quite large as compared with the typical hadronic scales. Such droplets will eventually hadronize by emitting hadrons from the surface. This multi-source emission mechanism should lead to strong non-statistical fluctuations of observables (see below). In refs. $[19,20]$ the evolution of individual droplets was studied numerically within a hydrodynamical approach including dynamical chiral fields (Chiral Fluid Dynamics). It has been demonstrated that the energy released at the spinodal decomposition can be directly transferred into the collective oscillations of the $(\sigma, \pi)$ fields which give rise to the soft pion radiation.

The above presented arguments apply for the case of a first order deconfinement phase transition as expected at high baryon densities. Recently in ref. [21] we have demonstrated that similar fragmentation phenomenon can occur in a system with crossover type of the phase transformation, if the bulk viscosity becomes large in the transition region. As follows from the lattice calculations [22], such situation can be expected in the QCD. This means that at $T \sim T_{c}$ the system suddenly becomes very stiff so that it cannot expand uniformly any more and breaks into pieces like a glass. The piece size in this case is determined by the condition that the collective expansion energy is fully dissipated due to the viscosity forces.

\section{Anomalous multiplicity fluctuations}

After the QGP break-up the Q droplets recede from each other according to the global expansion, predominantly along the beam direction. Hence their center-of-mass rapidities $y_{i}$ are in one-to-one correspondence with their spatial positions. Presumably $y_{i}$ will be distributed more or less evenly between the target and projectile rapidities. Since rescatterings in the dilute H phase 
between the droplets are rare, most hadrons produced from individual droplets will go directly into detectors. This may explain why freeze-out parameters extracted from the hadronic yields are always very close to the phase transition boundary [6].

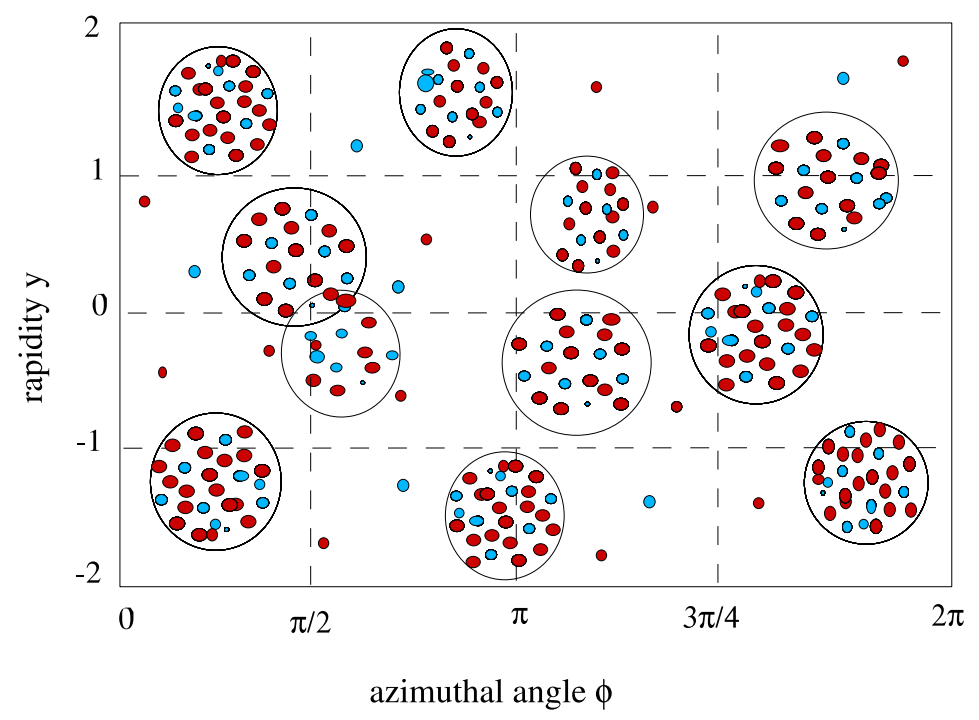

Figure 6: (Color online) Schematic view of the momentum space distribution of secondary hadrons produced from an ensemble of droplets. Each droplet emits hadrons (mostly pions) within a rapidity interval $\delta y \sim 1$ and azimuthal angle spreading of $\delta \phi \sim 1$.

In the droplet phase the mean number of produced hadrons in a given rapidity interval is $\langle N\rangle=\sum_{i}^{N_{D}} \overline{n_{i}}=\langle n\rangle\left\langle N_{D}\right\rangle$, where $\overline{n_{i}}$ is the mean multiplicity of hadrons emitted from a droplet $\mathrm{i}$, $\langle n\rangle$ is the average multiplicity per droplet and $\left\langle N_{D}\right\rangle$ is the mean number of droplets produced in this interval. If droplets do not overlap in the rapidity space, each droplet will give a bump in the hadron rapidity distribution around its center-of-mass rapidity $y_{i}[15,13,14]$. In case of the Boltzmann spectrum the width of the bump will be $\delta \eta \sim \sqrt{T / m}$, where $T$ is the droplet temperature and $m$ is the particle mass. At $T \sim 100 \mathrm{MeV}$ this gives $\delta \eta \approx 0.8$ for pions and $\delta \eta \approx 0.3$ for nucleons. Due to the radial expansion of the fireball the droplets should also be well separated in the azimuthal angle. The characteristic angular spreading of pions produced by an individual droplet is determined by the ratio of the thermal momentum of emitted pions to their mean transverse momentum, $\delta \phi \approx 3 T /\left\langle p_{\perp}\right\rangle \sim 1$. The resulting phase-space distribution of hadrons in a single event will be a superposition of contributions from different $\mathrm{Q}$ droplets superimposed on a more or less uniform background from the dilute $\mathrm{H}$ phase. Such a distribution is shown schematically in Fig. 6. It is obvious that such inhomogeneities (clusterization) in the momentum space will reveal strong non-statistical fluctuations of observables. The fluctuations will be more pronounced if primordial droplets are big, as expected in the Fair-Nica energy domain. If droplets as heavy as $15 \mathrm{GeV}$ are formed, each of them will emit up to $\sim 50$ pions within a narrow rapidity and angular intervals, $\delta \eta \sim 1, \delta \phi \sim 1$. If only a few droplets are produced in average per unit rapidity, $N_{D} \gtrsim 1$, they will be easily resolved and analyzed. On the other hand, the fluctuations 
will be suppressed by factor $\sqrt{N_{D}}$ if many small droplets shine into the same rapidity interval, as is expected at high collision energies.

It is convenient to characterize the fluctuations by the scaled variance $\omega_{N} \equiv\left(\left\langle N^{2}\right\rangle-\langle N\rangle^{2}\right) /\langle N\rangle$. Its important property is that $\omega_{N}=1$ for the Poisson distribution, and therefore any deviation from unity will signal a non-statistical emission mechanism. As shown in ref. [23], for an ensemble of emitting sources (droplets) $\omega_{N}$ can be expressed in a simple form,

$$
\omega_{N}=\omega_{n}+\langle n\rangle \omega_{D}
$$

where $\omega_{n}$ is an average multiplicity fluctuation in a single droplet, $\omega_{D}$ is the fluctuation in the droplet size distribution and $\langle n\rangle$ is the mean multiplicity from a single droplet. Since $\omega_{n}$ and $\omega_{D}$ are typically of order of unity, the fluctuations from the multi-droplet emission are enhanced by the factor $\langle n\rangle$. According to the picture of a first order phase transition advocated above, this enhancement factor could be as large as $\sim 10$. It is clear that the nontrivial structure of the hadronic spectra will be washed out to a great extent when averaging over many events. Therefore, more sophisticated methods of the event sample analysis should be applied as e.g. measuring event-byevent fluctuations in the hadron multiplicity distributions in a varied rapidity bin.

Finally it should be mentioned that up to now no significant effects in fluctuation observables have been found (see e.g. ref. [24]). Possible explanation is that the search was done at too high bombarding energies, when the expansion rate is too high to see traces of the phase transition. The future hopes are associated with experimental studies at lower bombarding energies, in the range of 10-40 AGeV. There are at least two arguments in favor of such strategy. First, such collisions will bring matter into the domain of high baryon densities where the first order deconfinement phase transition is predicted. Second, the produced matter will expand less violently, so that the predicted QGP droplets will be large enough to produce observable effects.

\section{Conclusions}

- Hydrodynamic modeling is very useful tool for understanding complicated dynamics of heavy-ion collisions at relativistic energies;

- In equilibrium scenario manifestations of the deconfinement phase transition are rather weak. Non-equilibrium effects like clusterization of the QGP and its direct conversion into hadrons may help to identify this phase transition;

- Strong non-statistical fluctuations of observables associated with the QGP droplets represent a very promising signal of the deconfinement phase transition;

- Low energy program at RHIC and future FAIR-NICA experiments will certainly help to find the onset of deconfinement and identify signals of the quark-gluon plasma.

I thank my colleagues A.V. Merdeev and L.M. Satarov for many fruitful discussions and help in the preparation of this talk. This work was supported in part by the DFG Grant 436 RUS 113/711/0-2 (Germany), and by the Grants NS-3004.2008.2 and RFFI 09-02-91331 (Russia). 


\section{References}

[1] L.M. Satarov, M.N. Dmitriev, and I.N. Mishustin, Phys. Atom. Nucl.72, 1390-1415 (2009).

[2] Particle Data Group, C. Amsler et al., Phys. Lett. B667, 1 (2008).

[3] S. Wheaton and J. Cleymans, hep-ph/0407174.

[4] Yu.B. Ivanov, A.S. Khvorostukhin, E.E. Kolomeitsev, V.V. Skokov, V.D. Toneev, and D.N. Voskresensky, Phys. Rev. C 72, 025804 (2005).

[5] D.H. Rischke, M.I. Gorenstein, H. Stöcker, and W. Greiner, Z. Phys. C 51, 485 (1991).

[6] A. Andronic, P. Braun-Munzinger, and J. Stachel, Nucl. Phys. A772, 167 (2006).

[7] H.W. Barz, B.L. Friman, J. Knoll, and H. Schulz, Phys. Rev. D40, 157 (1989).

[8] O. Scavenius, A. Mocsy, I.N. Mishustin, and D.H. Rischke, Phys. Rev. C64 (2001) 045202

[9] L.D. Landau, Izv. Akad. Nauk, ser. fiz. , 17, 51 (1953).

[10] J.D. Bjorken, Phys. Rev. D 27,140 (1983).

[11] D.H. Rischke, Y. Pürsün, J.A. Maruhn, H. Stöcker, and W. Greiner, Heavy Ion Phys. 1, 309 (1995).

[12] A.V. Merdeev, L.M. Satarov, I.N. Mishustin, paper in preparation.

[13] I.N. Mishustin, Phys. Rev. Lett. 82, 4779 (1999).

[14] I.N. Mishustin, Fluctuations near the deconfinement phase transition boundary. In Proc. of International Conference New Trends in High-Energy Physics: Experiment, Phenomenology, Theory (Yalta, Crimea, Ukraine, 10-17 Sep 2005), eds. Tomas Cechak, Laszlo Jankovsky, Iurii Karpenko, published in "Nuclear Science and Safety in Europe" (Springer, 2006), pp. 99-111; hep-ph/0512366.

[15] L.P. Csernai, I.N. Mishustin, Phys. Rev. Lett. 74, 5005 (1995).

[16] O. Scavenius, A. Dumitru, E. S. Fraga, J. T. Lenaghan and A. D. Jackson, Phys. Rev. D 63, 116003 (2001).

[17] Jorgen Randrup, Phys. Rev. Lett. 92, 122301 (2004).

[18] L.P. Csernai and I.N. Mishustin, work in progress (see L.P. Csernai's talk at this conference).

[19] I.N. Mishustin, O. Scavenius, Phys. Rev. Lett. 83, 3134 (1999).

[20] K. Paech, H. Stöcker and A. Dumitru, Phys. Rev. C 68, 044907 (2003).

[21] Giorgio Torrieri, Boris Tomasik, Igor Mishustin, Phys. Rev. C 77, 034903 (2008).

[22] F. Karsch, D. Kharzeev, K. Tuchin, arXiv0711.0914 [hep-ph].

[23] G. Baym, H. Heiselberg, Phys. Lett. B469, 7 (1999).

[24] V.P. Konchakovski, Mark I. Gorenstein, Elena L. Bratkovskaya. Phys. Rev. C76, 031901 (2007). 